\title{
Mapeamento do fluxo de trabalho das atividades em engenharia clínica: a experiência do Hospital das Clínicas da Faculdade de Medicina de Ribeirão Preto - Universidade de São Paulo
}

\section{Mapping of Workflow in Clinical Engineering: The experience of the University Hospital, Faculty of Medicine of RibeirãoPreto, University of São Paulo}

André FMM. Gomes ${ }^{1}$; Gabriela G Faustino; Monique Tonani ${ }^{1}$; Sídney Porcincula ${ }^{1}$; Sílvio C Somera ${ }^{1}$; Wilker Beicker², Antônio Pazin-Filho ${ }^{3}$

\begin{abstract}
RESUMO
A incorporação de equipamentos médicos hospitalares e outros recursos tecnológicos com alto valor agregado aumentam os desafios para realizar uma gestão eficiente e de qualidade pelas Organizações de Saúde. Nesse contexto, a utilização de ferramentas de apoio a gestão se torna essencial para o aproveitamento racional dos recursos de saúde, de forma a alcançar maior estabilidade e sustentabilidade do sistema como um todo. Assim, o presente trabalho teve como finalidade elaborar um mapeamento do fluxo de trabalho das atividades desenvolvidas no setor de Engenharia Clínica, responsável pela gestão de equipamentos médico diagnósticos do HCFMRP-USP, exposto na forma de fluxograma, objetivando posteriormente, sua incorporação e propagação dentre outros setores do hospital.
\end{abstract}

Palavras-chave: Engenharia Clínica; Mapeamento; Fluxo de Trabalho.

\begin{abstract}
The incorporation of medical devices and other technological resources with high aggregate value increases the challenges to achieve an efficient and quality management by Health Organizations. In this context, the use of tools to support the management becomes essential for the efficient use health resources in order to achieve greater stability and sustainability of the system as a whole. Thus, this study aimed to develop a mapping workflow of activities in the Clinical Engineering sector, responsible for managing medical diagnostic equipment at HCFMRP-USP, exposed in flow chart form, aiming later incorporation and spread among other hospital departments.
\end{abstract}

Key-words: Clinical Engineering; Mapping; Workflow.

1. Alunos do curso de pós-graduação Mestrado Profissionalizante - Gestão em Organizações de Saúde.

2. Coordenador do Serviço de Engenharia Clínica do Hospital das Clínicas da FMRP-USP.

3. Professor Associado II do Departamento de Clínica Médica da FMRP-USP e Coordenador da Unidade de Emergência do HCFMRP-USP.
Correspondência: Curso de Pós-graduação Mestrado Profissionalizante - Gestão em Organizações de Saúde da FMRP-USP. Aprovado para publicação em 14/08/2014 


\section{Introdução}

No atual cenário público de saúde, onde períodos de instabilidade política, econômica e social são variáveis constantes, e que o tornam ainda mais singular, temos observado com frequência os grandes desafios que os governos têm enfrentado para assegurar o acesso a esses serviços, principalmente após a criação do Sistema Único de Saúde (SUS) em 1988. A pluralização dos atendimentos e a garantia de sua realização à toda população, em todos os níveis de atenção, conforme os princípios de universalidade, equidade e integralidade fizeram com que o cenário da saúde ganhasse grande complexidade envolvendo o financiamento e suporte para a manutenção desses serviços.

Observamos de forma cada vez mais evidente a velocidade em que novas tecnologias vão surgindo, integrando muitos processos e serviços, e o quão dinâmico esse processo se mostra, sendo constantemente inovado, reinventado e se tornando cada vez mais aperfeiçoado às atuais exigências. ${ }^{1,2}$ Esse progresso técnico científico que vem revolucionando os tratamentos médicos e a assistência ao paciente, também tem se mostrado um dos principais problemas para os gestores de saúde. Com a incorporação de equipamentos médicos hospitalares e outros recursos tecnológicos com alto valor agregado, aumentaram também os desafios para realizar uma gestão eficiente e de qualidade.

Os departamentos de engenharia clínica e outras respectivas denominações presentes nos hospitais que são encarregados dessa função, sobretudo nos pertencentes aos níveis terciário e quaternário de atenção, constantemente se deparam com situações similares e comuns, ainda mais na área pública: a ineficiência na administração e gestão de recursos, agravado pela falta, ou, muitas vezes, incompatibilidade do repasse econômico necessário e da qualificação profissional dos recursos humanos que lá estão.

Dessa forma, a elaboração de propostas em potencial para alterar esse panorama são cada vez mais necessárias. Nesse sentido, alguns autores na literatura têm destacado a importância de atividades que promovam uma ampliação das informações ligadas aos processos e fluxo de trabalho nas organizações, como uma forma de integrar todos os envolvidos na cadeia de serviços, constituindo uma poderosa ferramenta para análise sistêmica do setor, e dessa forma, auxiliando na realização de uma gestão de excelência e transparência. ${ }^{3,4,5}$
Nesse contexto, o mapeamento do fluxo de trabalho se apresenta como uma ferramenta capaz de promover a visualização completa e conseqüente compreensão das atividades executadas no setor, os processos e departamentos envolvidos, facilitando e tornando mais simples a identificação de pontos de melhora nos serviços. ${ }^{6}$ De acordo com Soliman (1998), ${ }^{7}$ o mapeamento do fluxo de trabalho é uma técnica usada para detalhar os processos de negócios, focando os elementos importantes que influenciam o seu comportamento atual.

Por se tratar de um componente essencial para o gerenciamento e comunicação, o mapeamento pode ainda permitir a redução de custos na prestação de serviços, a redução nas falhas de integração entre sistemas e melhora do desempenho da organização, além de ser uma excelente ferramenta para possibilitar o melhor entendimento dos processos atuais e eliminar ou simplificar aqueles que necessitam de mudanças. ${ }^{8,3}$

Outro fator muito relevante no emprego do mapeamento das atividades é a instituição da transparência nas atividades, requisito muito valorizado pelas organizações, sejam públicas ou privadas. A importância de realizar uma gestão com transparência nos serviços é garantir a democratização do acesso às informações, demonstrando como são realizados os processos, a utilização dos recursos por uma organização, visando dessa forma desenvolver valores de integridade e ética. ${ }^{9}$ Segundo Schuster, Carpenter e Kane (1997), ${ }^{10}$ a transparência é definida como um conjunto de forças interativas que agem ao mesmo tempo envolvendo a alfabetização organizacional e financeira, e comunicação com linguagem fácil e acessível para todos.

Uma das grandes contribuições ao se instaurar um mapeamento do fluxo de atividades é o importante papel que essa ação proporciona, de desafiar os processos existentes, ajudando a formular uma variedade de perguntas críticas afim de encontrar possíveis gargalos, como em qual setor, área ocorrem mais frequente problemas, bem como identificar os respectivos responsáveis relacionados a essa questão., ${ }^{3,11}$

Resumidamente, os principais ganhos com o mapeamento do trabalho são ${ }^{12}$ :

- Maior conhecimento sobre os tempos e prazo de cada envolvido no serviço do equipamento;

- Identificação do local responsável pelo atraso/ não solução do equipamento;

- Redução de custos; 
- Ampliação da comunicação entre todos os envolvidos;

- Satisfação do cliente

- Transparência do serviço

Portanto, diante desse cenário, onde torna-se cada vez mais essencial o aproveitamento racional dos recursos de saúde, de forma a alcançar uma maior estabilidade e sustentabilidade do sistema de saúde como um todo é escopo desse projeto o desenvolvimento e mapeamento das atividades realizadas no setor de Engenharia Clínica do HCFMRP-USP e as unidades a ela relacionadas como forma de propor uma maior integração e transparência entre os envolvidos, propiciando a identificação dos locais /envolvidos em possíveis problemas dentro da divisão, e ainda, fomentar discussões em potencial para a busca de novas propostas com um foco na realização de uma gestão eficiente e transparente.

\section{Objetivos}

O objetivo deste projeto é caracterizar o mapeamento das atividades realizadas no Centro de Engenharia Clínica e Bioequipamentos do Hospital das Clínicas da Faculdade de Medicina de Ribeirão Preto - Universidade de São Paulo.

\section{Material e Métodos}

O presente trabalho é um estudo descritivo, estudo de caso, destinado a identificar e mapear os fluxos de trabalho (Workflow) existentes dentro do setor de Engenharia Clínica de um Hospital Universitário de nível terciário, objetivando auxiliar a gestão deste setor e também proporcionar uma maior transparência das atividades realizadas por este para os próprios funcionários e também os usuários do serviço prestado por estes.

O sistema de Workflow pode ser definido como tipo de Sistema de Informação, como a "automatização de um processo de negócio no sentido total ou parcial, durante a qual documentos, informações ou tarefas são passadas de um participante para outro, a fim de que sejam tomadas ações, de acordo com um conjunto de regras e procedimentos ${ }^{13}$.

O gerenciamento de um fluxo de trabalho deve apoiar os processos com suporte dos serviços de Engenharia e Tecnologia da Informação, destacadamente da seguinte forma: 1) Descrevendo os aspectos do processo que são relevantes para o controle e coordenação da execução de tarefas e 2) Auxiliando nos processos de mudança e implementação conforme as necessidades.

Por meio da utilização de workflows na organização é possível identificar os gargalos do serviço possibilitando melhor resultado nas tomadas de decisão. É necessário analisar o processo do trabalho, uma vez que a construção de workflow contribui não apenas para a gestão dos documentos, mas também para otimização dos processos.

As informações do presente trabalho foram obtidas através de entrevistas não estruturadas com o Coordenador da área e seis funcionários do setor de Engenharia Clínica do Hospital, subdivididos nas áreas, Apoio Administrativo, Apoio Tecnológico, Oficina de Bioequipamento e Oficina de Precisão.

Durante essas entrevistas os servidores relataram as atividades realizadas no setor e dentro de cada área englobada por este, sendo algumas vezes interrogados quando surgiam dúvidas. Cada entrevista teve duração média de 1 hora e 30 minutos e as visitas para realização destas foram realizadas durante o decorrer de um mês em diferentes dias, conforme disponibilidade da equipe do setor.

Após a realização das entrevistas foi realizada a análise do conteúdo destas a fim de esquematizar as atividades realizadas na área na forma de fluxograma, demonstrando os fluxos de trabalho. Para o desenho dos fluxogramas foi utilizado o programa Yed, que é de domínio público. O fluxograma obtido nesta etapa foram validados pelo coordenador do setor para garantir a exatidão do resultado obtido e está apresentado nos resultados deste trabalho.

Finalmente, com o fluxograma finalizado foi realizada uma análise objetivando verificar pontos falhos e que podem ser melhorados. Baseado nesta análise foram formulados algumas sugestões para ações futuras que visam otimizar o fluxo de trabalho envolvido neste setor.

\section{Resultados}

O mapeamento do fluxo de trabalho da Engenharia Clínica do HCFMRP, ilustrado através das figuras 1 e 2, corroborando com o que já foi exposto no presente trabalho, demonstra algumas características e critérios utilizados para o seu desenvolvimento. De acordo com a agência PNMSOFT (2014), ${ }^{14}$ dentre os 3 tipos de WorkFlow, o modelo sequencial, adotado 


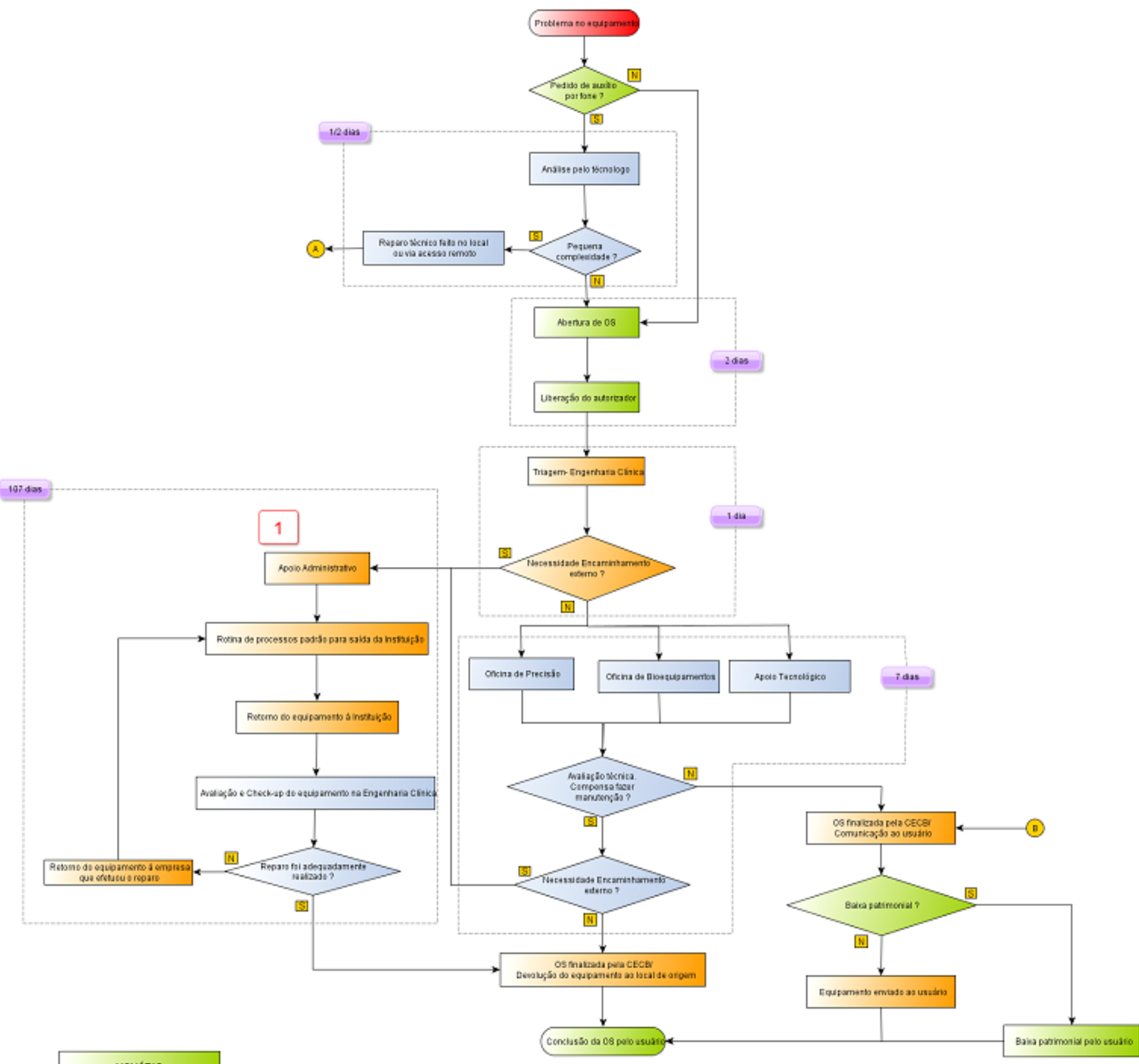

USUAR:O

APOIO ADMIN ENG.CLINICA

AREA TECMICA

ADNIN. HCIFAEPA

Figura 1: Mapeamento do fluxo das atividades no departamento de Engenharia Clínica do HCFMRP-USP. 


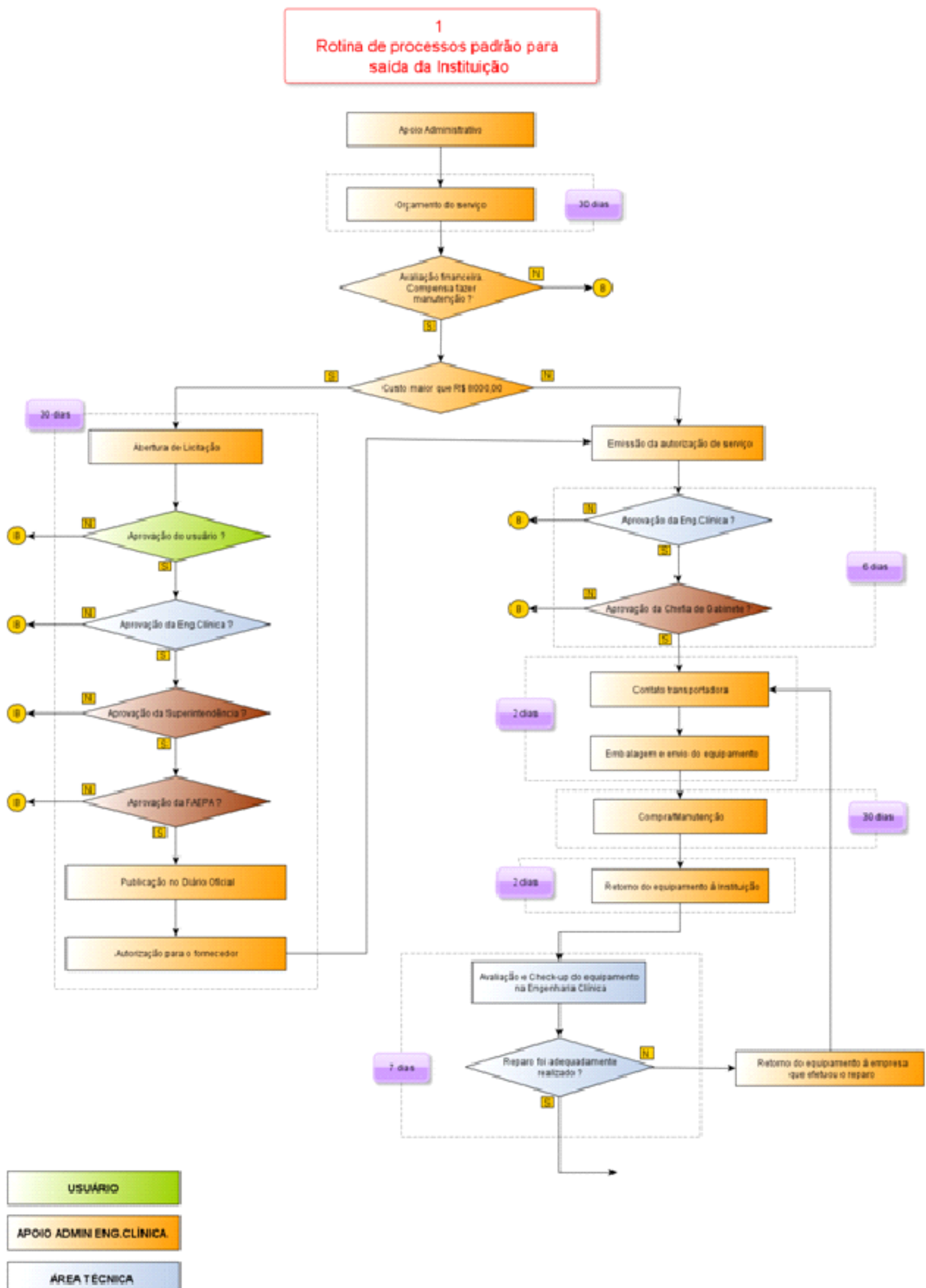

AREA TECNECA 
nesse trabalho, pode ser definido como sendo aquele construído baseado no progresso dos estágios das atividades.Para fins didáticos e de fácil visualização, as macro áreas do setor de Engenharia Clínica foram apresentadas sob uma escala de diferente cores, refletindo as atividades atribuídas a cada subdivisões: Usuário, Apoio Administrativo da Engenharia Clínica, Área Técnica e Administração HC/FAEPA.

A disposição e forma de apresentação desse fluxo de trabalho representou aspectos técnicos, normativos, políticos, discutidos e aprovados pelos envolvidos e responsáveis pelo setor.

\section{Discussăo}

A elaboração do mapeamento do fluxo de trabalho da Engenharia Clínica do HFMRP-USP proporcionou aos envolvidos uma série de benefícios, os quais podemos citar uma compreensão em escala macro do fluxo de informações e processos pelas suas diferentes subáreas, sendo ainda possível, a verificação dos responsáveis de cada etapa, além do prazo, um fator elementar observado na qualidade final do serviço, algo diretamente relacionado ao conceito de expectativa percebida pelo usuário - QPS. ${ }^{15}$ Nessa linha, Kmetz (2010) ${ }^{16}$ aponta que o mapeamento e definição do fluxo de trabalho é um fator essencial presente em programas de gestão da qualidade por apresentar como o fluxo de materiais e pessoas estão relacionados objetivando um mesmo propósito em comum.

Outra significativa vantagem do emprego do fluxo de trabalho, em uma escala micro, na Engenharia Clínica é, potencializar a eficiência na execução dos processos, uma vez que o esse mapeamento permite um maior acesso, clareza e disponibilidade à informação sobre as diversas etapas aos funcionários, facilitando ainda, ações de monitoramento e controle nas subdivisões do setor. ${ }^{17}$ Para a coordenação da Engenharia Clínica, esse mapeamento aliado a outras ferramentas gerenciais, pode resultar em facilidades para a gestão das atividades e tarefas, contribuindo como uma para a identificação e solução de problemas, sendo, portanto, valiosa fonte de apoio à tomada de decisão. ${ }^{4}$

A oportunidade de realizar e desenvolver esse projeto em conjunto com todos no setor, evidenciou a importância de tal atividade como elemento de estímulo do relacionamento e interação dos profissionais e coordenação. Essa ação se mostrou ainda mais rele- vante por propiciar um ambiente rico para a identificação e discussão de problemas, sugestões de ideias e opiniões, funcionando implicitamente como uma sessão de brainstorming.

Como resultados dessas reuniões, conseguimos elencar alguns fatores, os quais se mostraram relevantes para a otimização do trabalho realizado pelo setor de Engenharia Clínica do HCFMRP. À fim de facilitar a via de execução e buscando minimizar o tempo gasto para autorização do serviço, entendemos que, como toda a verba investida no setor provem da FAEPA e que em seu estatuto há possibilidade de compra direta sem licitação com valores até $\mathrm{R} \$ 20.000,00$ (Vinte mil reais), se mostra, portanto, plausível e factível, a possibilidade de execução de uma nova portaria do HC onde houvesse autorização para compras até esse valor.

Ainda, a atualização e introdução de informações no sistema de OS com a finalidade de comtemplar o trabalho total demandado pelos profissionais do setor, desde o primeiro momento na análise do problema dos equipamentos realizada pelos tecnólogos. Tal medida se mostra importante para a coordenação estimar e controlar o tempo gasto nas diferentes etapas do serviço, bem como garantir maior precisão nos prazos estabelecidos, e ainda, maior transparência para o usuário final.

Possivelmente alguns dos fatores limitantes desse estudo podem estar relacionados ao acesso e à acurácia de algumas informações e características técnicas obtidas referentes aos serviços realizados pela Engenharia Clínica. É de conhecimento que, algumas variáveis do trabalho são fortemente dependentes de fatores internos e externos, e ainda, difíceis de serem controladas, como por exemplo o prazo dos serviços. Ainda, algumas informações de aspectos quantitativos sobre o parque tecnológico de equipamentos e demanda, citados do trabalho, foram estimados, uma vez que não havia uma plena gestão e controle disponíveis em base de dados.

\section{Conclusões}

Assim, dentro do escopo proposto para este trabalho que era auxiliar e proporcionar maior transparência do fluxo de trabalho da Engenharia Clínica para os usuários do serviço, bem como para os funcionários envolvidos no setor, o presente trabalho conseguiu buscar soluções factíveis para melhorar a qualidade dos serviços realizados. A aplicação de ferra- 
mentas analíticas e gráficas de fácil manuseio e interpretação, amplamente citado na literatura em questão, mostra ser uma forma de tornar acessível e claro, informações sobre processos e fluxo de informações em uma Organização. Dessa forma, espera-se também que, a replicação das ideias e metodologias aqui utilizadas possam ser empregadas nas demais áreas do hospital, bem como todo o complexo HCFMRP. Por fim, nós autores gostaríamos de realçar as oportunidades que o desenvolvimento desse estudo nos propiciou. A discussão, as ideias e propostas abordadas, bem como a interdisciplinaridade entre discentes, docentes e profissionais em um hospital terciário, de ensino, com recursos humanos altamente qualificados, e toda a infraestrutura relacionada, propicia uma atmosfera rica para produção de novos conhecimentos e soluções nos mais diversos segmentos, algo fundamental para o suporte à uma gestão mais eficiente e de excelência.

\section{Agradecimentos}

Os autores expressam aqui o reconhecimento aos profissionais do Centro de Engenharia Clínica e Bioequipamentos do Hospital das Clínicas da Faculdade de Medicina de Ribeirão Preto - USP, que a partir das entrevistas concedidas, contribuíram para a realização deste trabalho.

\section{Referências}

1. BRASIL. Ministério da Saúde. Secretaria-executiva. Área de Economia da Saúde e Desenvolvimento. Avaliação de tecnologias em saúde: Ferramentas para a Gestão do SUS. Brasília : Editora do Ministério da Saúde, 2009.

2. Sullivan R, Peppercorn J, Sikora K, Zalcberg J, Meropol NJ, Amir $\mathrm{E}$, et al. Delivering affordable cancer care in high-income countries. Lancet Oncol. 2011; 12: 933-80.

3. Hunt, VD. Process mapping: how to reengineer your business processes. 1sted. New York: John Wiley \&Sons, Inc.; 1996.
4. Georgakopoulos D, Hornick M. An Overview of Workflow Management: From Process Modeling to Workflow Automation Infrastructure. DistribParallelDatabases. 1995; 3:119-53.

5. Pinho AF, Leal F, Montevechi JAB, Almeida DA. Combinação entre as técnicas de fluxograma e mapa de processo no mapeamento de um processo produtivo. XXVII Encontro Nacional de Engenharia de Produção,Foz do Iguaçu, PR; 2007. p.1-12.

6. Correia KSA, Almeida DAA. Aplicação da técnica de mapeamento de fluxo de processo no diagnóstico do fluxo de informações da cadeia cliente-fornecedor. XXII Encontro Nacional de Engenharia de Produção, Curitiba - PR; 2002. p. 1-8.

7. Soliman F. Optimum level of process mapping and least cost business process re-engineering. Int $\mathrm{J}$ Oper Prod Manag. 1998; 18: 810-16.

8. Villela CSS. Mapeamento de Processo como Ferramenta de Reestruturação e Aprendizado Organizacional.[Dissertação de Mestrado]. Florianópolis, Universidade Federal de Santa Catarina; 2000.

9. Tristão G. Transparência na administração pública. VII Congreso Internacional del CLAD sobre la Reforma del Estado y de laAdministración Pública, Lisboa- Portugal; 2002. p. 1-11.

10. Schuster JP, Carpenter J, Kane MP. O Poder do Gerenciamento Transparente. 1st ed. São Paulo: Futura, 1997; 305 p.

11. Johansson HJ, Mchugh P.Processos de negócios. São Paulo: Pioneira, 1995.

12. Harrington J. Aperfeiçoando processos empresariais. 1st ed. São Paulo: Makron Books, 1993. 343p.

13. Thom LH, lochpe C, Vicari D, Gus I. Processo de Desenvolvimento de Sistemas de Workflow Considerando Fatores Humanos e a Análise da Dinâmica Organizacional. International Symposium on Knowlegment Management / Document Management, ISKM-DM, Curitiba - PR; 2002. [capturado 8 out. 2013]. Disponívelem: http://www.inf.ufrgs.br/ lucineia/Papers/ ISKDM\%272000.pdf

14. Pnmsoft. What is a Workflow? [Internet]. [capturado 19 out. 2013]. Disponível em: http://www.pnmsoft.com/resources/bpmtutorial/workflow-tutorial/

15. Ruggeri R. Elementidi logistica industriale. Milano: CUSL, 1999.

16. Kmetz JL. Mapping Workflows and Managing Knowledge: Simply, Sensibly, Flexibly, and without Software. 2010. [capturado 2 nov. 2013]. Disponível em: http://www.buec.udel.edu/ kmetzj/business_consulting/WFMA\%20brief.pdf

17. Chaffey D. Groupware, Workflow and Intranets, Reengineering the Enterprise with Collaborative Software.1st ed. Woburn: Digital Press, 1998. 